\title{
AVALIAÇÃO DA EFICÁCIA, SEGURANÇA E TOLERABILIDADE DE RABEPRAZOL NO TRATAMENTO DE DOENÇAS ÁCIDO-PÉPTICAS
}

\author{
José Alves de FREITAS ${ }^{1}$, Lúcia Maria Praciano LIMA², \\ José Luiz RANIERI ${ }^{2}$, Cláudio OLIVIERI Jr. ${ }^{2}$, \\ Hélio José FRAGOSO ${ }^{2}$ e Décio CHINZON ${ }^{3}$
}

RESUMO - Racional - Rabeprazol sódico é um derivado benzimidazólico com potente ação inibidora da bomba de prótons. Aprovado na maioria dos países do mundo, inclusive no Brasil, a substância tem se mostrado muito eficaz para o tratamento das doenças ácido-pépticas, especialmente a doença por refluxo gastroesofágico. Objetivos - Avaliar a eficácia e tolerabilidade de rabeprazol em pacientes com esofagite por refluxo e/ou úlcera péptica gástrica e/ou duodenal na prática clínica, bem como avaliar o tempo necessário para a obtenção de alívio dos sintomas. Pacientes/Métodos - Foram avaliados 171 pacientes que procuraram consulta com gastroenterologista e que tiveram como diagnóstico a doença por refluxo ou úlcera péptica. Todos os pacientes eram ambulatoriais, de ambos os sexos, com idade superior a 18 anos. O diagnóstico, assim como o controle póstratamento foi obtido através do exame endoscópico. Todos os pacientes receberam $20 \mathrm{mg}$ de rabeprazol ao dia por 4 a 8 semanas, dependendo do diagnóstico e a critério do médico. Os pacientes receberam um diário para registro do início do alívio dos sintomas. Os dados obtidos no inicio e ao final do tratamento foram analisados através do teste não-paramétrico de Wilcoxon para amostras pareadas. Resultados - Dos 171 pacientes iniciais 162 completaram o estudo. Destes, $78(48,1 \%)$ tiveram como diagnóstico a doença por refluxo. Onze $(6,8 \%)$ apresentaram associação desta com úlcera duodenal e $7(4,3 \%)$ associado à úlcera gástrica. Em $39(24,1 \%)$ o diagnóstico foi de úlcera duodenal. A úlcera gástrica isoladamente estava presente em $24(14,8 \%)$ e associada à úlcera duodenal em $3(1,9 \%)$ casos. O tratamento foi de 4 semanas em $94,2 \%$ dos pacientes. Apenas 7 necessitaram 6 semanas e 3 foram tratados por 8 semanas. Mais da metade dos pacientes obtiveram alívio dos sintomas após o 1 dia de tratamento e após 7 dias, 89,5\% estavam totalmente assintomáticos. O índice de cicatrização das lesões foi de $88,3 \%$ dos casos. Vinte e sete pacientes $(15,2 \%)$ relataram eventos adversos leves que não necessariamente estavam relacionados à medicação, sendo os mais frequientes: diarréia, cefaléia e tonturas. Conclusões - O rabeprazol mostrouse altamente eficaz para o alívio dos sintomas de pacientes com doenças ácido-pépticas. Da mesma forma, a droga proporcionou altos índices de cicatrização das lesões. O medicamento mostrou ser seguro, com efeitos colaterais transitórios e sem maiores conseqüências para os pacientes.

DESCRITORES - Benzimidazóis. Bombas de próton. Úlcera péptica.

\footnotetext{
Faculdade de Medicina de Catanduva, SP. ${ }^{2}$ Gastroenterologista da cidade de São Paulo. ${ }^{3}$ Disciplina de Gastroenterologia Clínica da Faculdade de Medicina da Universidade de São Paulo, São Paulo, SP.

Endereço para correspondência: Dr. José Alves de Freitas - FAMECA - Coordenadoria de Graduação - Av. São Vicente de Paulo, 14545 - $15809-145$ - Catanduva, SP. e-mail: jalves@zup.com.br
} 


\section{INTRODUÇÃO}

Rabeprazol é um inibidor de bomba de prótons (IBP) de última geração, aprovado pelo Food and Drug Administration (FDA) para tratamento de doenças ácido-pépticas, incluindo: doença por refluxo gastroesofágico (DRGE), úlcera gástrica (UG), úlcera duodenal (UD) e síndrome de Zollinger-Ellison. Assim como os demais IBPs, essa droga tem se mostrado eficaz e segura ${ }^{(2)}$.

Estudos clínicos demonstraram que o medicamento é altamente eficaz para o alívio dos sintomas e cicatrização da esofagite por refluxo e de úlceras gástricas e duodenais ${ }^{(10)}$. Estudos comparativos utilizando-se $20 \mathrm{mg}$ de rabeprazol ao dia, versus placebo, durante 8 semanas, em pacientes com DRGE, o índice de cicatrização foi de $84 \%$ e $12 \%$ respectivamente ${ }^{(3)}$. Outro estudo clínico também envolvendo pacientes com DRGE, comparou rabeprazol $20 \mathrm{mg} /$ dia com ranitidina $150 \mathrm{mg} 4$ vezes ao dia, por 8 semanas. Os índices de cicatrização foram de $92 \%$ com rabeprazol e de $66 \%$ no grupo ranitidina $^{(7)}$. Estudo multicêntrico internacional comparando rabeprazol $20 \mathrm{mg} /$ dia com omeprazol $20 \mathrm{mg} / \mathrm{dia}$, também por 8 semanas, observaram índices de cicatrização de $92 \%$ e $94 \%$, respectivamente ${ }^{(11)}$.

Múltiplos estudos confirmaram não só a eficácia, mas também a segurança do rabeprazol, permitindo sua aprovação pelo FDA.

\section{Objetivos}

Primário: avaliar a eficácia e tolerabilidade de $20 \mathrm{mg}$ de rabeprazol administrado $1 \mathrm{vez}$ ao dia, em pacientes com esofagite por refluxo, UG e/ou UD ativas, na prática clínica de consultórios de gastroenterologistas de São Paulo.

Secundário: avaliar o tempo necessário para alívio dos sintomas, assim como a segurança e tolerabilidade do rabeprazol.

\section{Desenho do estudo}

Estudo multicêntrico, aberto, não-comparativo, realizado em consultórios de gastroenterologistas da cidade de São Paulo em pacientes com diagnóstico de DRGE, UG e/ou UD ativas.

\section{MATERIAL E MÉTODOS}

\section{Material}

Foram estudados 171 pacientes que procuraram um consultório de gastroenterologista e tiveram diagnóstico de DRGE, UG e/ou UD.

\section{Métodos}

A - Critérios de inclusão:

- Pacientes ambulatoriais, de ambos os sexos, com idade superior a 18 anos.

- Se do sexo feminino ou pré-menopausadas, com vida sexual ativa, deveriam estar em uso de método anticoncepcional.

- Apresentarem diagnóstico de esofagite por refluxo de graus I, II, ou III da classificação de Savary-Miller ou UG e/ou UD.

$B$ - Critérios de exclusão:

- Esofagites secundárias a doenças sistêmicas, tais como: esclerodermia, ingestão de cáusticos entre outras.

- Presença de varizes de esôfago ou gástricas.

- História de cirurgia gástrica ou esofágica prévia.

- Evidência endoscópica de sangramento gastrointestinal ou suspeita clínica de síndrome de Zollinger-Ellison.

- Mulheres grávidas ou lactantes.

- Enfermidade concomitante (passada ou presente) que pudesse dificultar a avaliação do estudo, tais como: doenças malignas, disfunção cardiovascular, renal ou hepática.

- Uso de corticosteróides, antiinflamatórios não-esteróides ou anti-neoplásicos.

- Abuso de drogas, álcool ou qualquer outra condição que pudesse interferir na aderência do paciente ao tratamento.

O diagnóstico foi estabelecido através de exame endoscópico.

Todos os pacientes utilizaram rabeprazol na dose de $20 \mathrm{mg}$ pela manhã. Todos foram alertados para não mastigar ou triturar o comprimido, devendo ser deglutido por inteiro.

O tempo de tratamento foi estabelecido pelo pesquisador de acordo com o diagnóstico: DRGE - 4 a 8 semanas, UG - 3 a 6 semanas, e UD - 2 a 4 semanas.

Os pacientes foram avaliados, no mínimo, duas vezes (pré e póstratamento). Consultas adicionais poderiam ser realizadas caso necessário. Os pacientes receberam um diário para registro do início do alívio dos sintomas.

\section{Análise Estatística}

Para comparação entre os dados obtidos na visita inicial e final utilizou-se o teste não-paramétrico de Wilcoxon para amostras pareadas. O nível de significância adotado foi de $5 \%$. 


\section{RESULTADOS}

\section{Pacientes Estudados}

Foram incluídos 171 pacientes dos quais 162 completaram o estudo, $78(48,1 \%)$ com diagnóstico de DRGE, sendo $56(71,8 \%)$ esofagite Grau I, $20(25,6 \%)$ grau II e $2(2,6 \%)$ grau III de acordo com a classificação de Savary-Miller. Onze pacientes $(6,8 \%)$ apresentaram ER associada à UD e $7(4,3 \%)$ associada à UG. Trinta e nove pacientes $(24,1 \%)$ tiveram diagnóstico de UD, 24 (14,8\%) UG e 3 (1,9\%) UG e UD associadas (Tabela 1).

TABELA 1 - Diagnósticos

\begin{tabular}{lcc}
\hline Avaliação Inicial & $\mathrm{n}$ & $\%$ \\
\cline { 2 - 3 } Esofagite por refluxo (I, II, III) & 78 & 48,1 \\
Esofagite + UD & 11 & 6,8 \\
Esofagite + UG & 7 & 4,3 \\
UD & 39 & 24,1 \\
UG & 24 & 14,8 \\
UD + UG & 3 & 1,9 \\
Total & 162 & 100 \\
\hline
\end{tabular}

\section{Dados Demográficos}

A idade média dos pacientes foi de 42,5 anos, variando de 18 a 80 anos. A altura média foi de $166,8 \mathrm{~cm}$ e o peso médio $71,4 \mathrm{~kg}$. Quanto ao sexo, $55 \%$ eram do sexo masculino e $45 \%$ do feminino. Oitenta e um por cento dos pacientes eram da raça branca (Tabela 2).

TABELA 2 - Dados demográficos

\begin{tabular}{llc}
\hline Característica & & \\
\cline { 1 - 1 } Sexo & M & $55 \%$ \\
& F & $45 \%$ \\
Raça & Branca & $81 \%$ \\
& Negra & $6 \%$ \\
& Mulata & $11 \%$ \\
Idade $(\mathrm{anos})$ & Oriental & $2 \%$ \\
Altura $(\mathrm{cm})$ & média \pm DP & $42,5 \pm 14,0$ \\
Peso $(\mathrm{kg})$ & média \pm DP & $166,8 \pm 8,8$ \\
& média \pm DP & $71,4 \pm 13,6$ \\
\hline
\end{tabular}

\section{Enfermidades Concomitantes}

Quarenta e nove pacientes $(28,2 \%)$ apresentavam enfermidades concomitantes ao iniciarem o tratamento com rabeprazol, sendo a hipertensão arterial a mais freqüente.

\section{Avaliação dos Sintomas}

Os sintomas: pirose diurna e noturna, regurgitação ácida, eructação, dor epigástrica diurna e noturna, naúseas e vômitos, foram avaliados de acordo com a seguinte escala de escores:

0 -ausente

1 - leve (o paciente percebe o sintoma, mas não necessita medicação)

2 - moderado (melhora prontamente com medicamentos habituais. Ex. antiácidos).

3 - grave (incomoda muito e não melhora com os medicamentos corriqueiros).

Os sintomas foram avaliados no pré e pós-tratamento em todos os pacientes e os dados obtidos comparados estatisticamente.

\section{Pirose Diurna}

Setenta e oito virgula oito por cento dos pacientes apresentavam pirose diurna no início do tratamento. Ao final do mesmo, 84,4\% estavam livres desse sintoma. $\mathrm{O}$ resultado foi altamente significativo (Gráfico 1).

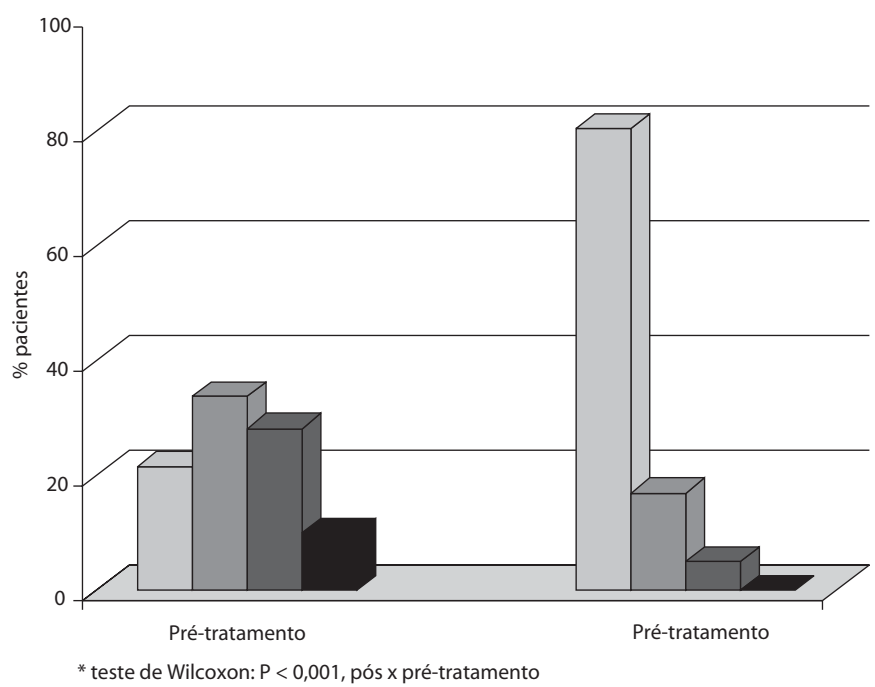

GRÁFICO 1 - Pirose diurna

\section{Pirose Noturna}

A pirose noturna estava presente em $67,3 \%$ dos pacientes no período pré-tratamento e em somente $14,6 \%$ ao final (Gráfico 2).

\section{Eructação}

Eructação estava presente em $62,6 \%$ dos pacientes antes do tratamento e ausente em $76,8 \%$ no pós-tratamento, com alta significância estatística (Tabela 3). 


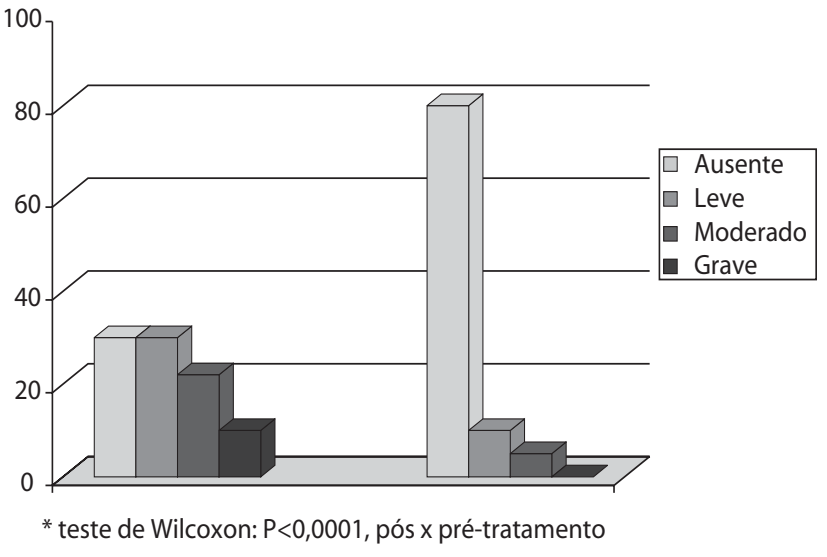

GRÁFICO 2 - Pirose noturna

\section{Regurgitação ácida}

Dos pacientes incluídos no estudo, $59,1 \%$ apresentavam regurgitação ácida antes do tratamento. Ao final do estudo, $84,8 \%$ estavam assintomáticos (Tabela 3 ).

\section{Dor epigástrica diurna}

A dor epigástrica diurna foi relatada por $84,8 \%$ dos pacientes antes do tratamento e apenas $14 \%$ continuavam a se queixar ao final. Do ponto de vista estatístico, a melhora foi significativa (Tabela 3).

\section{Dor epigástrica noturna}

Houve, também, melhora significativa da dor epigástrica noturna que estava presente em $71,3 \%$ dos pacientes antes do tratamento e em $10,4 \%$ ao final (Tabela 3 ).

\section{Naúseas}

Cerca de metade dos pacientes $(46,2 \%)$ apresentava naúseas antes de iniciar o tratamento e ao término deste, apenas 3 pacientes $(1,8 \%)$ se queixavam deste sintoma (Tabela 3 ).

\section{Vômitos}

Trinta e três pacientes $(18,7 \%)$ queixavam-se de vômitos no início do tratamento e apenas $3(1,8 \%)$, continuavam com esta queixa ao final do estudo (Tabela 3).

\section{Duração do Tratamento}

O tratamento durou 4 semanas em $94,2 \%$ dos pacientes. Apenas sete pacientes $(4,1 \%)$ necessitaram tratamento por 6 semanas, e três $(1,8 \%)$ por 8 semanas. Lembramos que o tempo de tratamento era estabelecido pelo pesquisador, uma vez que o estudo visava observar o que ocorria na prática diária dos consultórios de gastroenterologistas.

\section{Início do Alívio dos Sintomas}

O alívio dos sintomas ocorreu em 53,7\% dos pacientes após o $1^{\circ}$ dia de tratamento. Após 7 dias de tratamento, observaram-se que $89,5 \%$ dos pacientes estavam assintomáticos.

\section{Taxa de Cicatrização}

A endoscopia de controle foi feita em 143 pacientes após o tratamento, havendo cicatrização em $88,3 \%$ dos casos. As taxas de cicatrização por diagnóstico estão na Tabela 4.

TABELA 4 - Taxa de cicatrização

\begin{tabular}{lc}
\hline Diagnóstico endoscópico & Taxa $(\%)$ \\
Esofagite por refluxo & 84,4 \\
Esofagite + UD & 90,0 \\
Esofagite + UG & 100,0 \\
UD & 90,6 \\
UG & 90,9 \\
UD + UG & 100,0 \\
\hline
\end{tabular}

TABELA 3 - Avaliação dos sintomas

\begin{tabular}{|c|c|c|c|c|c|c|c|c|c|c|c|c|}
\hline & \multicolumn{2}{|c|}{ Eructação* } & \multicolumn{2}{|c|}{ Disfagia* } & \multicolumn{2}{|c|}{ Vômitos* } & \multicolumn{2}{|c|}{ Náuseas* } & \multicolumn{2}{|c|}{$\begin{array}{c}\text { Dor } \\
\text { epigástrica } \\
\text { diurna* }\end{array}$} & \multicolumn{2}{|c|}{$\begin{array}{c}\text { Dor } \\
\text { epigástrica } \\
\text { noturna* }\end{array}$} \\
\hline Ausente & 37,4 & 76,8 & 77,2 & 95,1 & 81,3 & 98,2 & 53,8 & 94,5 & 15,2 & 86,0 & 28,7 & 89,6 \\
\hline Leve & 37,4 & 21,3 & 12,9 & 4,3 & 13,5 & 1,8 & 32,2 & 4,3 & 34,5 & 10,4 & 29,2 & 8,5 \\
\hline Total & 100,0 & 100,0 & 100,0 & 100,0 & 100,0 & 100,0 & 100,0 & 100,0 & 100,0 & 100,0 & 100,0 & 100,0 \\
\hline
\end{tabular}

* teste de Wilcoxon: $P<0,001$, final inicial 


\section{Eventos Adversos}

Vinte e sete pacientes $(15,2 \%)$ apresentaram eventos adversos, sendo os mais freqüentes: diarréia (5\%), cefaléia (3\%) e tontura ( $2 \%)$, que poderiam estar ou não relacionados à medicação.

\section{DISCUSSÃO}

Os IBPs constituem hoje, uma das classes de medicamentos mais usados mundialmente para o tratamento das doenças ácido-pépticas. O primeiro IBP foi lançado na Europa em 1988, durante um congresso de Gastroenterologia em Roma, e no ano seguinte nos Estados Unidos. Uma década após, verificou-se a expansão das indicações terapêuticas e pelo menos quatro drogas encontravam-se disponíveis no mercado: omeprazol, lansoprazol, pantoprazol e rabeprazol. A decisão de prescrever um ou outro IBP deve levar em conta a sua eficácia, segurança e custo.

Em meta análise envolvendo vários estudos clínicos, observaram-se que, para doses equivalentes, os IBPs apresentam eficácia e segurança equivalentes ${ }^{(9)}$. Entretanto, apesar de apresentarem estruturas químicas e mecanismo de ação semelhantes, existem diferenças farmacológicas significativas entre os IBPs. A mais marcante diz respeito ao tempo de ativação da droga no estômago. Em pH 1.2, todos os IBPs são ativados rapidamente (1 a 2 minutos), já em pH 5.1, este tempo é de 1.5 horas para o omeprazol e lansoprazol, e apenas 7 minutos para o rabeprazol $^{(8)}$. Outra diferença significativa ocorre em relação ao tempo de início de inibição da $\mathrm{H}^{+} \mathrm{K}$-ATPase. Cinco minutos após a ingestão de rabeprazol, observa-se significativa inibição da enzima, enquanto que o omeprazol e lansoprazol requerem 30-45 minutos para atingir o mesmo grau de inibição ${ }^{(1)}$.

Estudo clínico, duplo cego, cruzado, comparado a placebo, onde se avaliou o $\mathrm{pH}$ intragástrico de 23 pacientes $H$. pylori negativos, administrando-se rabeprazol, omeprazol e placebo, observou-se que 24 horas após a primeira dose da medicação, o tempo médio em que o $\mathrm{pH}$ ficou acima de 4,0 foi: de 44,1\% com o rabeprazol, de $24,7 \%$ para o omeprazol $(P<0,001)$ e de $7,6 \%$ para o placebo $(P=0.001)$. No $8^{\circ}$ dia, esses valores persistiam significativamente diferentes sendo: $60,3 \%$ para o rabeprazol versus $51,4 \%$ com rabeprazol $(P<0,001)$. Adicionalmente, os mesmos investigadores demonstraram que o rabeprazol provocou supressão ácida de $88,0 \%$ após a primeira dose, comparada a $42,0 \%$ com o omeprazol ${ }^{(12)}$.
Estudos clínicos, comparando-se a eficácia de rabeprazol e omeprazol para o alívio dos sintomas e cicatrização das lesões na UD, UG e na DRGE, utilizando-se doses padrão (20 mg), os índices de cicatrização da UD após 2 e 4 semanas foram semelhantes, com ligeira superioridade para o rabeprazol, no alívio dos sintomas após 4 semanas. Com relação à UG, os índices de cicatrização foram semelhantes tanto após 3, como após 6 semanas $^{(5)}$.

Da mesma forma, estudo multicêntrico europeu, avaliando o alívio dos sintomas e cicatrização das lesões em pacientes com esofagite erosiva demonstrou que tanto o rabeprazol, como o omeprazol foram igualmente eficazes tanto após 4 , como após 8 semanas $^{(6)}$.

Recentemente, minucioso estudo de meta análise, avaliando a relação custo-eficácia de diferentes IBPs para o tratamento de manutenção da esofagite erosiva, evidenciou que os índices de prevenção da recurrência de sintomas foram de $86 \%$ nos pacientes em uso de rabeprazol, $68 \%$ com lansoprasol e $81 \%$ com omeprazol, com média de custo por paciente (considerando-se hospitalização, procedimentos diagnósticos, consultas e preço da medicação) de 1.414 dólares americanos para rabeprazol, comparado a 1.671 com lansoprazol e 1.599 com omeprazol $^{(4)}$.

Finalmente, vale lembrar que a pesquisa de H. Pylori, embora tenha sido realizada na maioria dos pacientes, não foi objeto deste estudo, porque outro protocolo está em andamento analisando não só o papel desta bactéria, mas também possíveis recidivas ou falhas no tratamento.

\section{CONCLUSÕES}

A análise dos resultados obtidos neste estudo permite as seguintes conclusões:

O rabeprazol é medicamento altamente eficaz para o alívio sintomático de pacientes portadores de doenças ácido-pépticas. Neste estudo o desaparecimento dos sintomas ocorreu com apenas uma dose da medicação em mais da metade dos pacientes.

Da mesma forma, a droga proporciona altos índices de cicatrização da esofagite por refluxo nos seus diferentes graus.

Proporciona altos índices de cicatrização de úlcera péptica quer seja gástrica, ou duodenal.

Além de eficaz, o rabeprazol é medicamento seguro, já que raramente induz a efeitos colaterais e estes, quando ocorrem, são transitórios e sem maiores conseqüências para os pacientes. 
Freitas JA, Lima LMP, Ranieri JL, Olivieri Jr C, Fragoso HJ, Chinzon D. Efficacy, safety and tolerability of rabeprazole in treatment of acid-peptic diseases. Arq Gastroenterol 2002;39(1):60-5.

ABSTRACT - Background - Rabeprazole, a substituted benzimidazole, represents a new generation of proton pump inhibitors that has recently been approved by the FDA and European Union for treatment of acid-related diseases. Objectives - To assess the efficacy and tolerability of rabeprazole $20 \mathrm{mg}$ in actual conditions of use in everyday clinical practice on subjects with diagnosis of erosive gastroesophageal reflux disease and/or gastric and/or duodenal ulcer. Patients/Methods - A total of 171 outpatients (55\% men, 45\% women) with a mean age of 42.5 years were enrolled in this trial. The majority of subjects (81.0\%) were Caucasians. Patients with endoscopically confirmed erosive/ulcerative gastroesophageal reflux disease (Savary-Miller classification), duodenal ulcer and/or benign gastric ulcer were eligible to receive rabeprazole 20 $\mathrm{mg}$ once daily for 4 to 8 weeks, depending on the diagnosis and at investigators' discretion. Patients were requested to record their symptoms in a diary card in a daily basis. Results - One hundred and sixty two patients completed the study in accordance with the protocol. Reflux esophagitis was diagnosed in $78(48.1 \%)$ patients, duodenal ulcer in $39(24.1 \%)$ and gastric ulcer in $24(14.8 \%)$. Eleven (6.8\%) patients presented reflux esophagitis associated with duodenal ulcer and $7(4.3 \%)$ associated with gastric ulcer. Finally, 3 (1.9\%) presented both gastric and duodenal ulcer. Fifty-three percentage of patients were free of symptoms on the first day of treatment and $89.5 \%$ after a week. The healing rate was $84.4 \%$ for patients with reflux esophagitis, $90.6 \%$ for duodenal ulcer and $90.9 \%$ for gastric ulcer. The adverse effects were minimal and transitory. Conclusions - Rabeprazole is highly effective and well tolerated in acute healing of reflux esophagitis and peptic ulcers. In adittion, it provides fast symptoms relief .

HEADINGS - Benzimidazoles. Proton pumps. Peptic ulcer.

\section{REFERÊNCIAS BIBLIOGRÁFICAS}

1. Besancon M, Simon A, Sachs G, Shin JM. Sites of reaction of the gastric H, KATPase with extracytoplasmatic thiol reagents. J Biol Chem 1997;272:22438-46.

2. Chiba N. Proton pump inhibitors in acute healing and maintenance of erosive or worse esophagitis: a systematic overview. Can J Gastroenterol 1997;11 Suppl B:66B-73B.

3. Cloud ML, Olovich K. LY307640 versus placebo in healing erosive, ulcerative reflux esophagitis. Gastroenterology 1995;108 Suppl:73.

4. Dean BD, Siddique RM, Yamashita BD, Bhattacharjya AS, Ofman JJ. Costeffectiveness of proton-pump inhibitors for maintenance therapy of erosive reflux esophagitis. Am J Health-Syst Pharm 2001;58:1338-46.

5. Dekkers CP, Beker JA, Thjodleifsson B, Gabryelewicz A, Bell NE, Humphries TJ. Comparison of rabeprazole $20 \mathrm{mg}$ versus omeprazole $20 \mathrm{mg}$ in the treatment of active duodenal ulcer: a European multicentre study. Aliment Pharmacol Ther 1999; 13:179-86.

6. Dekkers CP, Beker JA, Thjodleifsson B, Gabryelewicz A, Bell NE, Humphries TJ. Double-blind, placebo controlled comparison of rabeprazole $20 \mathrm{mg}$ versus omeprazole $20 \mathrm{mg}$ in the treatment of erosive or ulcerative gastro-oesophageal reflux disease. The European Rabeprazole Study Group. Aliment Pharmacol Ther 1999; 13:49-57.
7. Humphries TJ, Spera A. Rabeprazole sodium (E3810) once daily is superior to ranitidine $150 \mathrm{mg}$ qid in the healing of erosive or ulcerative gastroesophageal reflux disease. Gastroenterology 1996;110 Suppl:4.

8. Kromer W, Krüger U, Huber R, Hartmann M, Steinijans VW. Differences in pHdependent activation rates of substituted benzimidazoles and biological in vitro correlates. Pharmacology 1998;56:57-70.

9. Kromer W, Horbach S, Lülmann R. Relative efficacies of gastric proton pump inhibitors: their clinical and pharmacological basis. Pharmacology 1999;59:5777.

10. Stach WA, Knifton A, Thirlwell D, Cockayne A, Jenkins D, Hawkey CJ, Atherton JC. Safety and efficacy of rabeprazole in combination with four antibiotic regimens for the eradication of Helicobacter pylori in patients with chronic gastritis with or without peptic ulceration. Am J Gastroenterol 1998;93:1909-13.

11. Thjodleifsson B, Dekkers CPM. Rabeprazol sodium $20 \mathrm{mg}$ once daily is similar to omeprazole $20 \mathrm{mg}$ once daily in the treatment of erosive or ulcerative GERD. Gastroenterology 1997;112 Suppl:A312.

12. Williams MP, Sercombe J, Hamilton MI, Punder RE. A placebo-controlled trial to asses the effects of a 8 days of dosing with rabeprazole versus omeprazole on 24-h intragastric acidity and plasma gastrin concentrations in young healthy male subjects. Aliment Pharmacol Ther 1998;12:1079-89.

Recebido em 3/10/2001 Aprovado em 14/1/2002

\section{Médicos participantes do estudo:}

Alberto Youssef Laham

Chiemi Nakazato

Eduardo Kutchell de Marco

Elaine Fernandez

Eliana R. Cardoso

Elmir de Souza C. Filho

Elpídio Cassaro Júnior

Fabio G. B. de Carvalho

Flavio Martins de Souza

Francisco P. Pagliaro

Frederico Augusto da S. Almeida
Jorge Kazuo Nakauchi

José Carlos Vilela

José Henrique G. Brochado

Julio Cesar Caporale

Leonaldo Lagatta Benatti

Luís Kazuo Ogawa

Manoel Antônio C. de Azevedo

Mário Hiroyuki Egami

Nelson Fontana Margarido

Nora Ney Paes

Paulo A. Berchielli
Pedro Wolf Oksman

Rafael Munhoz Plana

Renee Fioravanti Landi

Roberto Ananias

Roberto Louzano

Rodrigo A. J. D. Abraldez

Sandra Frugis

Sandra R. Franceschini

Sandra Regina Vesco

Silvio Gabor 